Article

\title{
In-Depth Analysis of the Effect of Fragmentation on the Crystallization-Driven Self-Assembly Growth Kinetics of 1D Micelles Studied by Seed Trapping
}

\author{
Gerald Guerin ${ }^{1,2, *(D)}$, Paul A. Rupar ${ }^{3}$ and Mitchell A. Winnik ${ }^{2,4, *}$ \\ 1 Shanghai Key Laboratory of Advanced Polymeric Materials, Key Laboratory for Ultrafine Materials of \\ Ministry of Education, School of Materials Science and Engineering, \\ East China University of Science and Technology, Shanghai 200237, China \\ 2 Department of Chemistry, University of Toronto, 80 St. George Street, Toronto, ON M5S 3H6, Canada \\ 3 Department of Chemistry, University of Alabama, Tuscaloosa, AL 35487, USA; parupar@ua.edu \\ 4 Department of Chemical Engineering and Applied Chemistry, University of Toronto, \\ Toronto, ON M5S 3E2, Canada \\ * Correspondence: gguerin@ecust.edu.cn (G.G.); mwinnik@utoronto.ca (M.A.W.)
}

check for updates

Citation: Guerin, G.; Rupar, P.A.; Winnik, M.A. In-Depth Analysis of the Effect of Fragmentation on the Crystallization-Driven Self-Assembly Growth Kinetics of 1D Micelles Studied by Seed Trapping. Polymers 2021, 13, 3122. https://doi.org/ $10.3390 /$ polym 13183122

Academic Editors: Holger Schmalz and Volker Abetz

Received: 26 August 2021

Accepted: 10 September 2021

Published: 16 September 2021

Publisher's Note: MDPI stays neutral with regard to jurisdictional claims in published maps and institutional affiliations.

Copyright: (c) 2021 by the authors. Licensee MDPI, Basel, Switzerland. This article is an open access article distributed under the terms and conditions of the Creative Commons Attribution (CC BY) license (https:// creativecommons.org/licenses/by/ $4.0 /)$.

\begin{abstract}
Studying the growth of 1D structures formed by the self-assembly of crystalline-coil block copolymers in solution at elevated temperatures is a challenging task. Like most 1D fibril structures, they fragment and dissolve when the solution is heated, creating a mixture of surviving crystallites and free polymer chains. However, unlike protein fibrils, no new nuclei are formed upon cooling and only the surviving crystallites regrow. Here, we report how trapping these crystallites at elevated temperatures allowed us to study their growth kinetics at different annealing times and for different amounts of unimer added. We developed a model describing the growth kinetics of these crystallites that accounts for fragmentation accompanying the 1D growth process. We show that the growth kinetics follow a stretched exponential law that may be due to polymer fractionation. In addition, by evaluating the micelle growth rate as a function of the concentration of unimer present in solution, we could conclude that the micelle growth occurred in the mononucleation regime.
\end{abstract}

Keywords: block copolymers; crystallization-driven self-assembly; kinetics; fragmentation; growth

\section{Introduction}

"Seeing is believing" is a well-known idiom that has found an echo in science, where many breakthroughs involved microscopy techniques. In polymer science, one of the major discoveries was made in 1957 by Andrew Keller [1], while studying homopolymer single crystals by transmission electron microscopy (TEM). Keller confirmed that the thin lamellae of these crystals consisted of folded chains, as first suggested by Storks [2], and then showed that the lamellar thickness of a homopolymer single crystal depends directly on the temperature at which it was grown [3]. Since that time, microscopy techniques have become an important tool to further investigate crucial aspects of polymer crystallization, e.g., crystal morphology [4-7] and crystal growth kinetics [8-11].

Several groups have examined the self-assembly in solution of crystalline-coil block copolymers (BCPs) that crystallize to form 1-dimensional (1D) micelles [12-18]. In several cases, the length and the composition of these micelles can be controlled precisely by adding free block copolymer ("unimer") with the same crystalline block, leading to the formation of elongated micelles or more complex structures referred to as block co-micelles [19-21]. Among these BCPs, those with a polyferrocenyldimethylsilane (PFS) core-forming block have been the most intensely studied. PFS BCPs have allowed the formation of the most advanced structures via stepwise hierarchical assembly [22-28]. Such sophisticated structures are, however, difficult to create with other crystalline-coil BCPs, a situation 
compounded by our limited fundamental understanding of the crystallization and growth of these 1D micelles.

To follow the growth kinetics of homopolymer single crystals in solution by microscopy, the crystals need to be isolated from their supersaturated unimer solution at different annealing times. For 2D single crystals, one can sediment the crystals [29] or transfer a crystal suspension quickly from one thermostated bath to another to surround the original growth front with a crystalline layer of different thickness [8]. For 1D core crystalline micelles, Boott et al. [11] followed the growth kinetic of PFS- $b$-PDMS (where PDMS stands for polydimethylsiloxane) core-crystalline micelles by depositing a drop of the micelle solution onto a TEM grid at a given annealing time and measuring the micelle lengths. They evaluated the effect of solvent, unimer concentration and block ratio on the micelle growth rate of PFS- $b$-PDMS micelles at temperatures where micelle fragmentation and dissolution could be ignored.

Furthermore, valuable information about the formation and growth of core-crystalline micelles can be obtained by studying their growth kinetics as a function of polymer concentration. Indeed, crystal growth in solution is considered to be interface-controlled, and the growth-determining step is the attachment of straight-chain segments ("stems") to the growth front and their rearrangement to form a surface nucleus [30-33]. Modern theory identifies different growth regimes depending on the number and rate at which stems spread on the crystal face $[29,34]$. These regimes are predicted to exhibit different dependences on polymer concentration, $c$, and can be identified experimentally by studying the polymer concentration dependence of crystal growth rates, $G$. This relationship is given by $G \propto c^{\gamma}$, where $\gamma$ is the concentration exponent. For polymer single crystals, $\gamma$ ranges from 0.2 to 2 [8,35]. A value of $\gamma$ lower than 1 implies the presence of a barrier to chain deposition at the crystal growth front, while $\gamma$ larger than 1 suggests cooperation between several unimers in solution to form a stable nucleus that would grow on the crystal face.

More recently, we have been particularly interested in understanding the main factors that affect micelles dissolution [36,37]. We noticed that when a seed solution was heated at a temperature where most of the seed dissolved, the surviving seeds could broaden and extend [38]. To further investigate this phenomenon, here we report the growth kinetics study of core-crystalline micelles in the presence of different amounts of unimer, at a temperature where the micelles could both fragment and dissolve. For this purpose, we pre-heated solutions of $\mathrm{PFS}_{53}-b-\mathrm{PI}_{637}$ (where PI stands for polyisoprene, and the subscripts represent the degree of polymerization of each block) crystallites at $75{ }^{\circ} \mathrm{C}$, and added different amounts of unimer of the same BCP. After different annealing times, we injected a large excess of $\mathrm{PFS}_{60}-b$-PDMS 660 to trap the growing crystallites and measured their length for each annealing time, following a seed-trapping approach developed previously [36]. In-depth analysis of the lengths of the trapped seeds and that of the control samples (without trapping the seeds) allowed us to develop a kinetic model that accounts for the fragmentation of the seeds during their growth. We also showed that the growth kinetics could be well described by a stretched exponential, in agreement with the kinetics study of Boott et al. [11]. To explain these results, we hypothesize that the stretched exponential is caused by polymer fractionation. Finally, from the concentration dependency of the seed growth rates, we showed that the $1 \mathrm{D}$ growth occurs via the successive addition of polymer chains to the exposed crystal faces.

\section{Materials and Method}

Decane (99+\%) and Karstedt's catalyst were purchased from Sigma-Aldrich (Oakville, $\mathrm{ON}$, Canada) and used without further purification.

The $\mathrm{PFS}_{53}-b-\mathrm{PI}_{637}\left(\mathrm{M}_{\mathrm{n}}, \mathrm{GPC}=56,300, \mathrm{D}=1.01\right)$ and $\mathrm{PFS}_{60}-b-\mathrm{PDMS}_{660}$ were synthesized by one of us and have been reported in ref [39]. 


\subsection{Transmission Electron Microscopy}

Bright-field transmission electron microscopy (TEM) images were taken at the nanoimaging facility of the chemistry department of the University of Toronto using a Hitachi H-7000 instrument (Hitachi High-Tech Corporation, Tokyo, Japan). Samples were prepared by placing one drop of solution on a Formvar carbon-coated grid, touching the edge of the droplet with a filter paper to remove excess liquid and allowing the grid to dry.

For each sample, micelle length distributions were determined by tracing more than 200 micelles using the software ImageJ (NIH, Laboratory for Optical and Computational Instrumentation, LOCI, University of Wisconsin, Madison, WI, US). Error bars were calculated using the standard error of the mean, s.e.m., obtained with a $99 \%$ confidence interval.

\subsection{Sample Preparation}

Six vials, each containing $4 \mathrm{~mL}$ of the same seed solution $(\mathrm{c}=0.02 \mathrm{mg} / \mathrm{mL})$ were heated at $75^{\circ} \mathrm{C}$ in a heating bath. After $40 \mathrm{~min}$ of heating, different aliquots $(0,16,33,52$, 76 and $82 \mu \mathrm{L})$ of $\mathrm{PFS}_{53}-b-\mathrm{PI}_{637}$ unimer heated in decane $(\mathrm{c}=4.8 \mathrm{mg} / \mathrm{mL})$ at $100{ }^{\circ} \mathrm{C}$ were added to these solutions. Those solutions were then further annealed at $75^{\circ} \mathrm{C}$ for 100,420 , 1200 and $2640 \mathrm{~min}$. After each annealing time, $1 \mathrm{~mL}$ of each solution was transferred into empty vials that were also pre-heated to $75^{\circ} \mathrm{C}$. Half $(0.5 \mathrm{~mL})$ of each of these solutions were then injected in an empty vial and let to cool to room temperature $\left(23^{\circ} \mathrm{C}\right)$, following the usual self-seeding procedure, while to the second half we added a 5 times excess of $\mathrm{PFS}_{60}-b-\mathrm{PDMS}_{660}$ unimers that was pre-heated in decane at $100{ }^{\circ} \mathrm{C}$. This second set of samples was briefly swirled and let at $75{ }^{\circ} \mathrm{C}$ for 5 more minutes to fully mix $\mathrm{PFS}_{60}-b$ $\mathrm{PDMS}_{660}$ with the $\mathrm{PFS}_{53}-b-\mathrm{PI}_{637}$ unimers remaining in solution. The samples were then removed from the heating bath to cool to room temperature. This procedure allowed us to trap the $\mathrm{PFS}_{53}-b-\mathrm{PI}_{637}$ surviving seeds with the large excess of $\mathrm{PFS}_{60}-b-\mathrm{PDMS}_{660}$ unimer. After two days of aging at room temperature, we added $0.5 \mathrm{~mL}$ of decane to each trapped seed solution, followed by $0.1 \mathrm{~mL}$ of Karstedt's catalyst. The samples were let to age one more day and then studied by TEM. In parallel, the control samples were aged at room temperature for two days prior to be imaged by TEM. This led to a total of 48 samples that were imaged by TEM.

Note that to follow the micelle growth kinetics as a function of unimer concentration, we used a $\mathrm{PFS}_{53}-b-\mathrm{PI}_{637}$ seed solution in decane prepared for a previous study [36] and that was carefully stored in a sealed container and aged for one year at $23^{\circ} \mathrm{C}$. Over this time, the size (number average length, $L_{\text {seed, } R T}$, equal to $43.5 \mathrm{~nm}$ ) and concentration of seed crystallites remained constant (Figure S1), but aging decreased the number of crystallites that dissolved at a given annealing temperature (Figure S2). We attribute the enhanced robustness of the one-year-old seeds to an increase in the crystallinity of the 1D PFS core. To confirm that this long aging time did not affect the mechanism of the seed dissolution, we plotted the length distribution of one-year aged seeds annealed and trapped at $75{ }^{\circ} \mathrm{C}$, and compared it with the length distribution of the freshly prepared seeds annealed and trapped in similar conditions (Figure S3).

\subsection{Definition of Key Parameters}

The parameter $p$ represents the ratio of the amount of $\mathrm{PFS}_{53}-b-\mathrm{PI}_{637}$ unimer added to the solution at $75{ }^{\circ} \mathrm{C}\left(m_{\text {uni,added }}\right)$ to the mass of seed crystallites present in the seed solution at $23{ }^{\circ} \mathrm{C}\left(m_{\text {seeds, } R T}\right)$ prior to heating the solution to $75^{\circ} \mathrm{C}$. $L_{t s}(p, t)$ is the number average length of the trapped seeds annealed at $75^{\circ} \mathrm{C}$ for different annealing times, while the number average length of the untrapped micelles cooled to $23^{\circ} \mathrm{C}$ is defined as $L_{\text {mic }}(p, t)$.

Seeded growth can be well described by a simple equation [19]:

$$
L_{\text {mic }}=\left(\frac{m_{\text {uni }}}{m_{\text {seed }}}+1\right) L_{\text {seed }}
$$


where $L_{\text {mic }}$ and $L_{\text {seed }}$ are the number average lengths of the micelles and the seeds, respectively, while $m_{\text {uni }}$ is the mass of unimer added to the solution and $m_{\text {seed }}$ is the mass of seeds. Despite its apparent simplicity, one can extract a large amount of information from Equation (1), helping us understand some key phenomena related to seeded growth and self-seeding.

\section{Results and Discussion}

The surviving seeds could easily be delineated by our seed-trapping protocol (Figure 1) [36]. Figure 1f,g shows representative TEM images of the stained trapped seeds after the solution was annealed at $75^{\circ} \mathrm{C}$, for $2640 \mathrm{~min}$ without unimer added $(p=0$, Figure 1f) and with the largest amount of $\mathrm{PFS}_{53}-b-\mathrm{PI}_{637}$ unimer added $(p=4.9$, Figure $1 \mathrm{~g})$.

Control

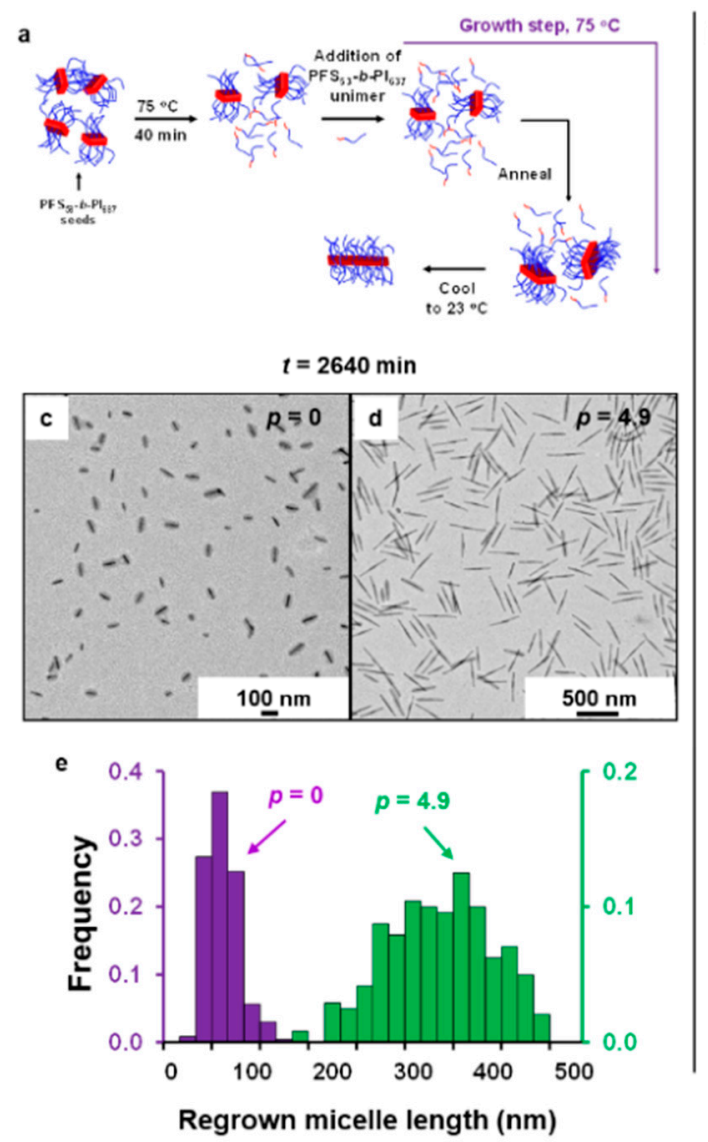

Trapped seeds

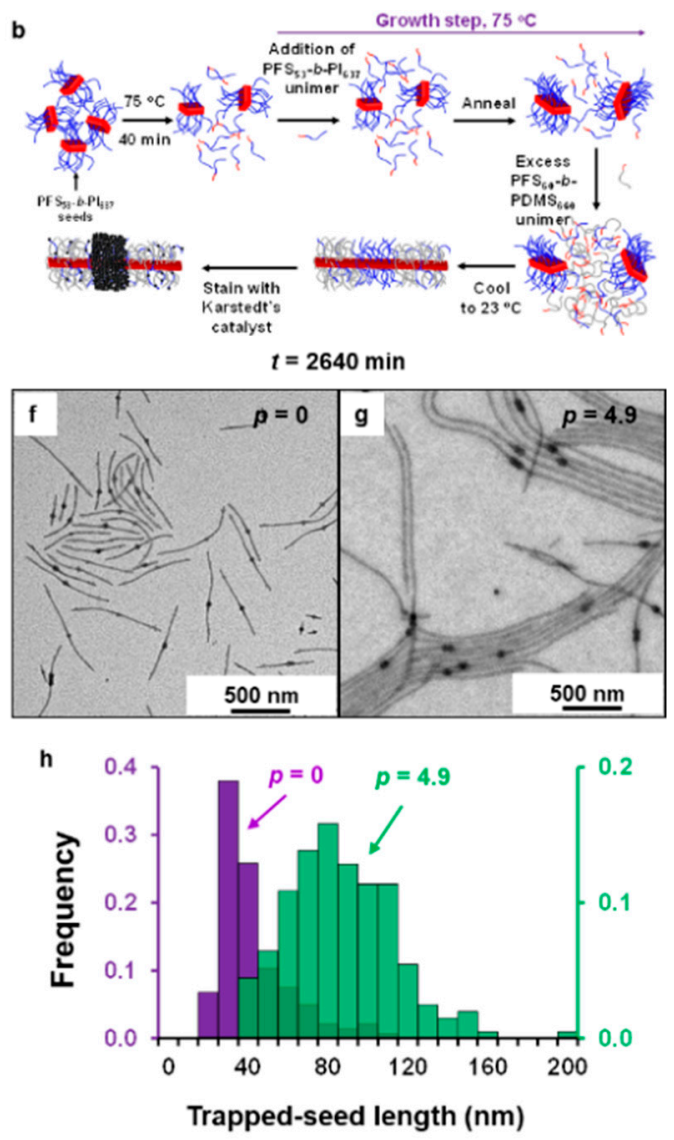

Figure 1. Seed growth kinetics studied by seed trapping. Schematic diagram describing (a) the control and (b) the seedtrapping experiments performed to study the growth kinetics of $\mathrm{PFS}_{53}-b-\mathrm{PI}_{637}$ crystallites annealed at $75{ }^{\circ} \mathrm{C}$ for different times in the presence of added $\mathrm{PFS}_{53}-b-\mathrm{PI}_{637}$ unimer. In this scheme we use a color code to represent different chemical species: Red represents polyferrocenyldimethylsilane (PFS) (either as the PFS block of a unimer or the crystalline core of a micelle), blue represents polyisoprene (PI), grey represents polydimethylsiloxane (PDMS), while the black spheres represent the platinum nanoparticles from the Karstedt's catalyst used to stain PI. (c,d) TEM images of PFS $53-b$-PI 637 micelles obtained by heating seeds at $75{ }^{\circ} \mathrm{C}$ for $2640 \mathrm{~min}$, and letting the solution cool to $23{ }^{\circ} \mathrm{C}$, (c) without $\mathrm{PFS}_{53}-b$ - $\mathrm{PI}_{637}$ unimer added $(p=0)$, and (d) in the presence of an initial mass ratio $(p=4.9)$ of $\mathrm{PFS}_{53}-b$ - $\mathrm{PI}_{637}$ unimer added to $\mathrm{PFS}_{53}-b$ - $\mathrm{PI}_{637}$ seeds. (e) Respective histograms of the length distributions of the regrown micelles without ( $p=0$, purple columns), and with unimer added ( $p=4.9$, green columns). (f,g) TEM images of $\mathrm{PFS}_{53}-b-\mathrm{PI}_{637}$ seeds trapped after 2640 min of annealing at $75^{\circ} \mathrm{C}$, (f) without $\mathrm{PFS}_{53}-b$-PI 637 unimer added $(p=0)$, and (g) in the presence of an initial mass ratio of $\mathrm{PFS}_{53}-b-\mathrm{PI}_{637}$ unimer added to $\mathrm{PFS}_{53}-b-\mathrm{PI}_{637}$ seeds, $p=4$.9. (h) Respective histograms of the length distribution of the surviving $\mathrm{PFS}_{53}-b-\mathrm{PI}_{637}$ seeds without ( $p=0$, purple columns), and with unimer added ( $p=4.9$, green columns). Samples (f,g) were stained with Karstedt's catalyst to highlight the PI rich regions. 
It is important to note that, as shown in a previous study [36], the trapped seeds are ca. $3.5 \mathrm{~nm}$ longer than the stained seeds as observed by TEM due to the shrinkage of the corona block induced by the cross-linking of the corona by the karsted's catalyst.

The effect of the addition of $\mathrm{PFS}_{53}-b-\mathrm{PI}_{637}$ unimer on $\mathrm{PFS}_{53}-b-\mathrm{PI}_{637}$ seeds can be seen in Figure 1f-h, in Figures S4-S15 and Tables S1 and S2. $L_{t s}(0,2640)$ is similar to that of the un-aged seeds trapped at room temperature (Figure S16), suggesting that the dissolution of some of the seeds did not induce an obvious growth of the surviving seeds at $75{ }^{\circ} \mathrm{C}$. In the presence of extra unimer $(p=4.9$, Figure $1 \mathrm{~g})$, and after the same annealing time (2640 $\mathrm{min}$ ), the length distribution of the seeds shifted to larger values, broadened and became Gaussian-like (Figure 1h). However, one sees that even after 2 days of annealing, the length of the trapped seeds remained much smaller than those of the corresponding control samples that were fully regrown at $23^{\circ} \mathrm{C}$ (Figure 1c-e), a clear indication that most of the unimer remained in solution at $75^{\circ} \mathrm{C}$.

Figure 2a shows the evolution of micelles that regrew after annealing (control experiments), $L_{\text {mic }}(p, t)$, as a function of annealing time for each amount of unimer added to the solution. For all the amounts of unimer added, one sees that the lengths of the micelles regrown at $23{ }^{\circ} \mathrm{C}$ decreased with annealing time. This result was rather surprising since one would intuitively expect the lengths of the micelles regrown at $23^{\circ} \mathrm{C}, L_{\text {mic }}(p, t)$ to be independent of annealing time. For each annealing time, however, the lengths of the micelles subsequently regrown at room temperature, $L_{\text {mic }}(p, t)$ versus $p$ obeys Equation (1), still increasedlinearly as a function of the amount of unimer present in solution (Figure $2 b$ ).
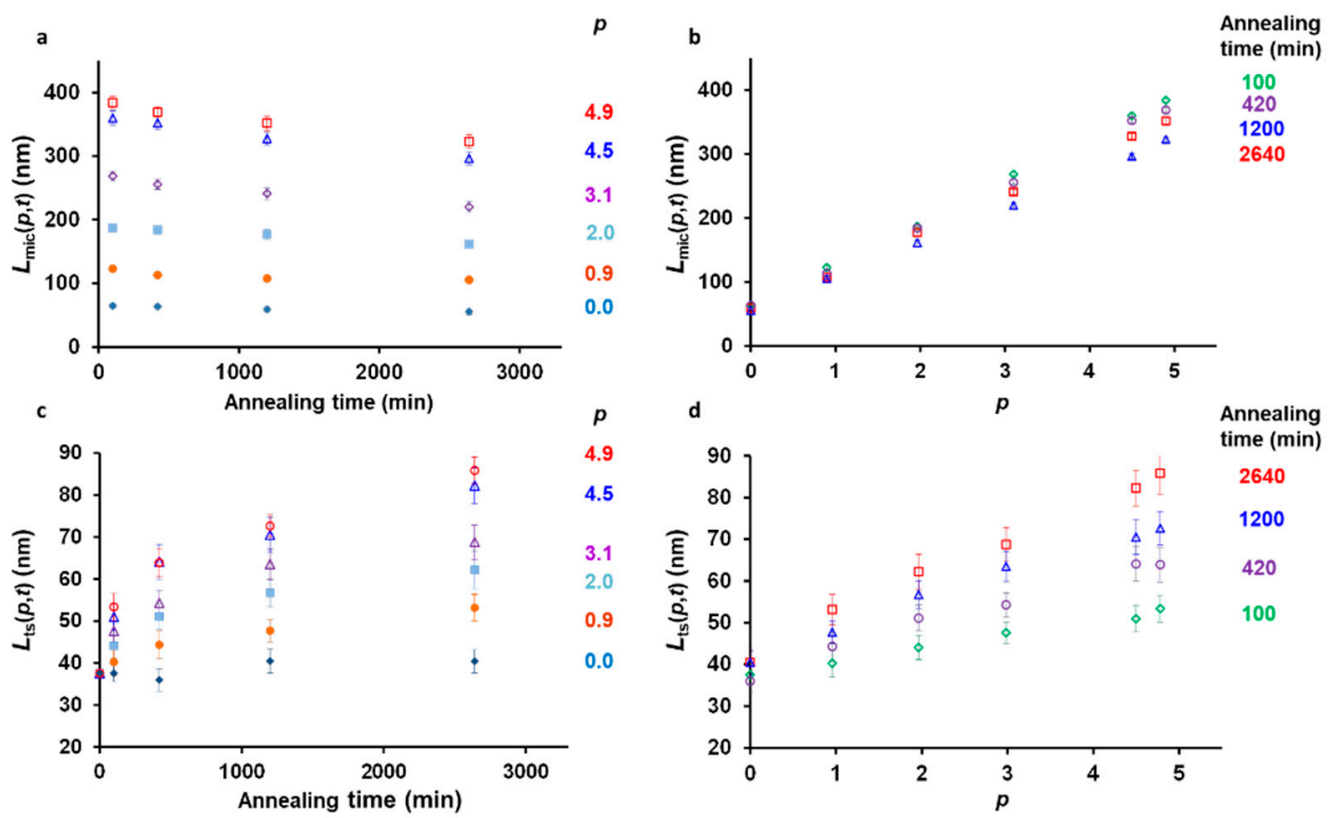

Figure 2. Evolution of the number average lengths of micelle annealed at $75{ }^{\circ} \mathrm{C}$ and regrown at room temperature solutions as a function of (a) time and (b) ratios $p$. Evolution of the number of average lengths of trapped seeds as a function of (c) time and (d) ratios $p . p$ is the ratio of the mass of unimer added to the hot seed solution, $m_{\text {uni,added }}$, to the mass of seeds originally present in the solution, $\mathrm{m}_{\text {seeds, } R T}$. Error bars correspond to the s.e.m. of the length distributions.

In contrast, the lengths of the seeds trapped at $75^{\circ} \mathrm{C}, L_{t s}(p, t)$ increased as a function of time (Figure 2c), following much more conventional behavior. Seeded growth was, however, extremely slow since even after two days of annealing, the trapped seeds remained much smaller than the micelles at room temperature, reaching only $90 \mathrm{~nm}$ for the largest amount of unimer injected in the solution. The growth kinetics were also non-linear, slowing down with time (Figure S17). Micelle growth, although extremely slow, was still noticeable since, for $p=4.9$, the trapped seeds were ca. twice longer after $2640 \mathrm{~min}$ of annealing than after $100 \mathrm{~min}$. Finally, in Figure $3 \mathrm{~d}$ we show the plot of the length of the 
trapped seeds as a function of the different amount of unimer added to the solution for each annealing time. In this plot, one observes that $L_{t s}(p, t)$ increased linearly as a function of $p$, as already seen for $L_{\text {mic }}(p, t)$ versus $p$.

a

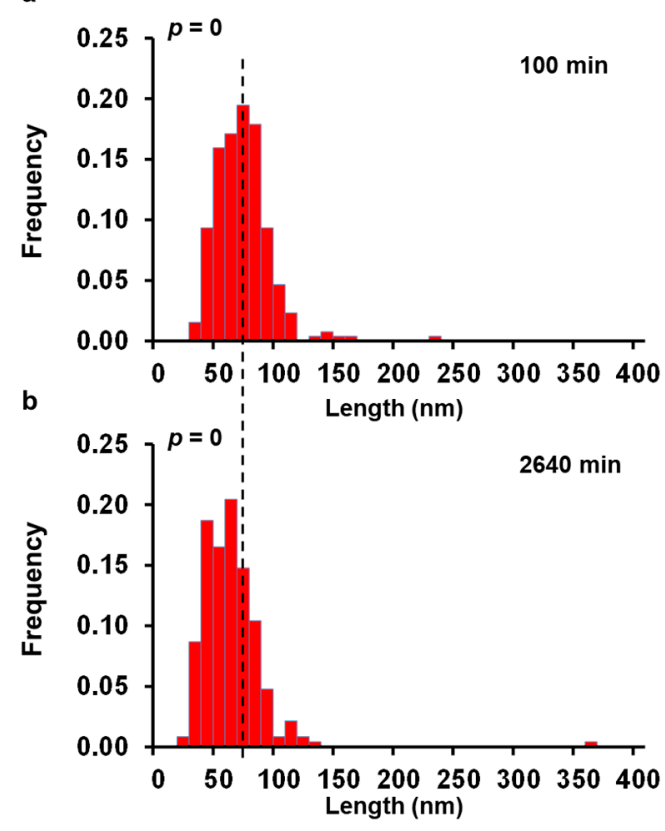

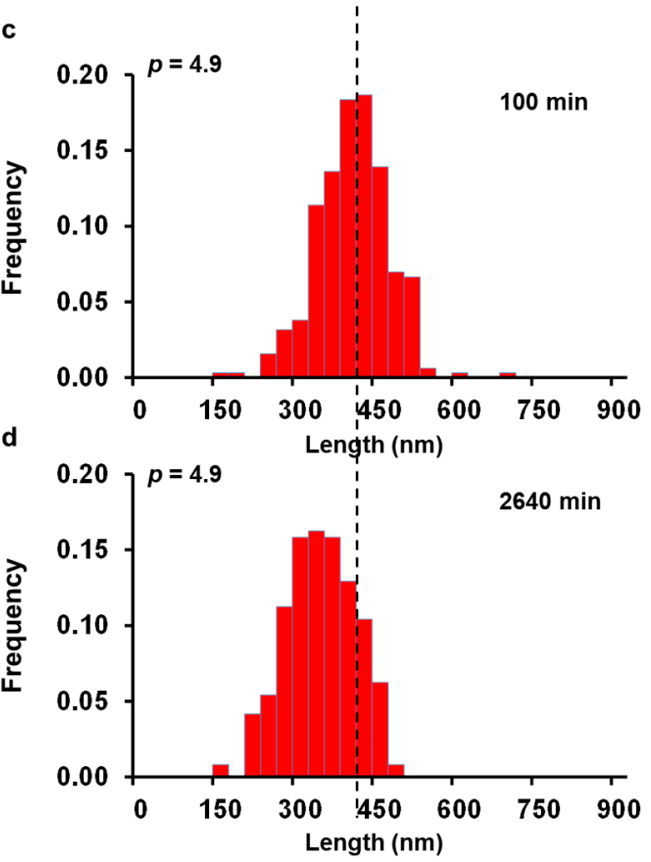

Figure 3. Histograms of the length distributions of $\mathrm{PFS}_{53}-b-\mathrm{PI}_{637}$ micelles regrown at room temperature for $p=0$ after (a) $100 \mathrm{~min}$, (b) $2640 \mathrm{~min}$, and for $p=4.9$ after (c) $100 \mathrm{~min}$ and (d) $2640 \mathrm{~min}$ of annealing at $75{ }^{\circ} \mathrm{C}$. The vertical dashed lines highlight the shift of the micelle length distributions towards shorter values.

The seed-trapping and control experiments were rather simple to perform, leaving little doubts about the validity of the results obtained. Interestingly, their combination coupled with the use of Equation (1) (which is also highly straightforward) can unravel complex phenomena, as shown in the following sections.

\subsection{Control Experiments: Lateral Growth versus Fragmentation during Annealing}

The number average lengths of the seeds annealed at $75^{\circ} \mathrm{C}$ for different times $(100,420$, 1200 and $2640 \mathrm{~min}$ ) (Figure S18) and cooled to room temperature decreased monotonically from ca. $64.4 \mathrm{~nm}$ for the sample annealed for $100 \mathrm{~min}$ down to $55.4 \mathrm{~nm}$ when annealed for $2640 \mathrm{~min}$. Although the length distributions of the sample shifted toward lower values (Figure $3 a, b$ ), the variation was quite small and could be considered, a priori, as part of the experimental error. However, similar behavior was observed for all the control samples, becoming increasingly noticeable as the amount of unimer added to the solution increased (Figure $3 c, d$ ), pointing toward a systematic effect of the annealing time on the final length of the micelles regrown at room temperature.

Previous works have shown that two main phenomena could explain this behavior:

(a) Unimer chains could add laterally onto the micelles during annealing. These chains would thus not participate to the elongation of the micelles. Since lateral growth is expected to be time dependent, the amount of unimer in solution that could participate to the elongation of the micelle at $23{ }^{\circ} \mathrm{C}$ would decrease with time.

(b) The micelles could fragment with time, increasing the number of seeds in solution which would lead to a decrease in the lengths of the micelles once they regrew at $23^{\circ} \mathrm{C}$.

The one-year aged seed crystallites were much more stable towards annealing at $75^{\circ} \mathrm{C}$ than their freshly prepared counterpart (Figure S2). Therefore, one can conclude that this long aging time favored the packing of the crystalline core and decreased the distance between two grafting points. As a result, the densification of the micelle crystalline core 
would lead to an increase in their grafting density, hindering the lateral growth of the micelles [38]. We thus assume that the decrease in $L_{\text {mic }}(p, t)$ as a function of annealing time is mainly due to seed fragmentation, while lateral growth can be neglected. We verified these assumptions by evaluating the ratio $L_{\text {mic }}(p, t) / L_{\text {mic }}(0, t)$ as a function of annealing time for both scenarios, and comparing it with the experimental plot of $L_{\text {mic }}(p, t) / L_{\text {mic }}(100, t)$ versus time shown in Figure 2b.

The lateral growth of the micelles during annealing would lead to an increase in the linear aggregation number of the micelles in the section that was regrown. Therefore, we can rewrite Equation (1) to express $L_{\text {mic }}(p, t)$ as a function of both $p$ and $t$ :

$$
L_{m i c}(p, t)=\left(\frac{N_{a g g / L}(p, t)}{N_{a g g / L} / R T} \frac{m_{u n i}(p, t)}{m_{t s}(p, t)}+1\right) L_{t s}(p, t)
$$

where $N_{\operatorname{agg} / L}(p, t)$, is the linear aggregation number of the trapped seeds, $N_{a g g / L, R T}$ is the linear aggregation number of the seeds at room temperature. $m_{u n i}(p, t)$ is the amount of unimer that is still present in solution after it was annealed at $75^{\circ} \mathrm{C}$ for a time $t$.

We recall that $m_{\text {seeds, } R T}=m_{\text {uni }}(0,0)+m_{t s}(0,0)$, while by definition, $m_{\text {added }}(p, 0)=p m_{\text {seeds, } R T}$, where $m_{\text {uni }}(0,0)$ is the mass of unimer coming from the dissolution of some of the starting seeds, $m_{\text {added }}(p, 0)$ is the mass of unimer added to the solution and $m_{t s}(0,0)$ is the mass of the surviving seeds just after heating. Equation (2) thus gives (see Supporting discussion, Section I):

$$
L_{\text {mic }}(p, t)=L_{t s}(0,0)\left[(p+1) \frac{m_{\text {seeds }, R T}}{m_{t s}(0,0)}\right]+L_{t s}(p, t)\left[1-\frac{N_{a g g / L}(p, t)}{N_{a g g / L, R T}}\right]
$$

For $t=0, N_{a g g / L}(p, 0)=N_{a g g / L, R T}$ (the linear aggregation number does not change), leading to:

$$
L_{\text {mic }}(p, 0)=\frac{m_{\text {seed, }, R T}}{m_{t s}(0,0)}(p+1) L_{t s}(0,0)
$$

Here, we note that for the specific case where $p$ and $t$ are equal to 0 , Equation (4) gives:

$$
\frac{m_{\text {seeds }, R T}}{m_{t s}(0,0)}=\frac{L_{m i c}(0,0)}{L_{t s}(0,0)}
$$

Therefore, Equation (3) becomes:

$$
\frac{L_{m i c}(p, t)}{L_{m i c}(p, 0)}=1-\frac{L_{t s}(p, t)}{L_{m i c}(0,0)}\left(\frac{N_{a g g}(p, t)-N_{a g g, R T}}{N_{a g g, R T}}\right) \frac{1}{(p+1)}
$$

On the other hand, if the decrease of $L_{m i c}(p, t)$ as a function of time is solely due to the fragmentation of the seeds as a function of time (and, thus to an increase in the number of seeds), we can show (Supporting discussion, Section II) that:

$$
L_{m i c}(p, t)=\frac{m_{\text {seed }, R T}}{m_{t s}(0,0)}(p+1) L_{t s, f}(p, t)
$$

where $L_{\mathrm{ts}, \mathrm{f}}(p, t)$ is the length of the fragmented seeds as a function of annealing time.

We note that Equation (7) is equivalent to Equation (4), using $L_{t s, f}(p, t)$ instead of $L_{t s}(p, t)$. At time, $t=0$, just after the seed solution reached $75{ }^{\circ} \mathrm{C}$, although the seeds may have fragmented, they did not grow, thus, the length of the fragmented seeds at $t=0$ is the same as the length of the trapped seeds, i.e., $L_{t s, f}(p, 0)=L_{t s}(p, 0)=L_{t s}(0,0)$. We can thus write:

$$
\frac{L_{m i c}(p, t)}{L_{m i c}(p, 0)}=\frac{L_{t s, f}(p, t)}{L_{t s}(0,0)}
$$


Equations (6) and (8) indicate that the evolution of $L_{m i c}(p, t) / L_{m i c}(100, t)$ versus time for different values of $p$ strongly depends on the annealing history. If lateral growth occurs during annealing (Equation (6)) then, one should expect $L_{m i c}(p, t) / L_{\text {mic }}(100, t)$ to vary with the amount of unimer added to the solution, while in the case of seed fragmentation (Equation (8)), $L_{\text {mic }}(p, t) / L_{\text {mic }}(100, t)$ would be independent of $p$ if the seed fragmentation does not depend on the amount of unimer added to the solution.

To evaluate which equation better describe the experimental results, we plotted $L_{\text {mic }}(p, t) / L_{\text {mic }}(100, t)$ versus time for the different amount of unimer added to the solution (Figure 4). In this plot, $L_{m i c}(p, t) / L_{m i c}(100, t)$ appears to be independent on the amount of unimer injected into the seed crystallite solution for the annealing times investigated, a strong indication that the change in the final lengths of the micelles for the control experiment is mainly due to seed fragmentation (additional discussion can be found in Supporting Information).

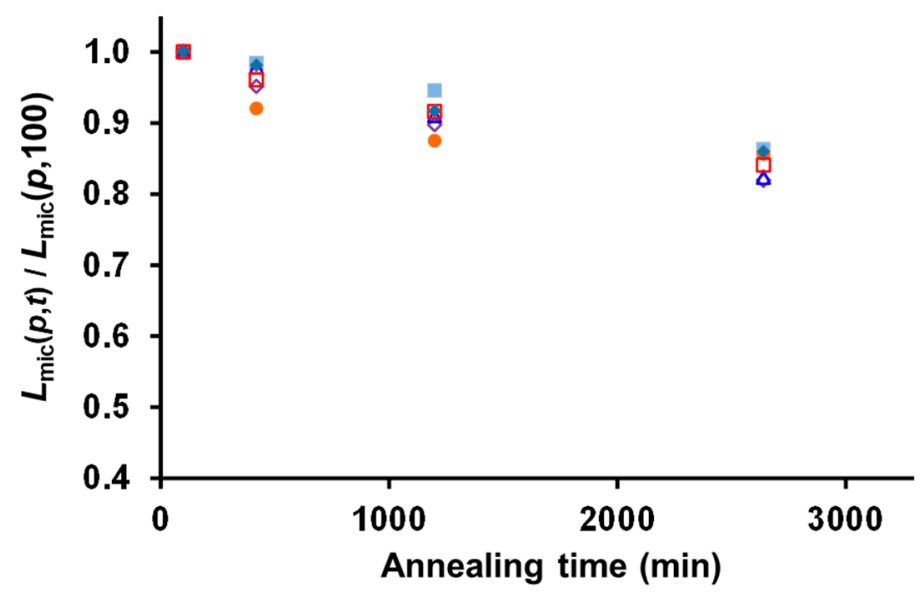

Figure 4. Plot of $\left[L_{\text {mic }}(p, t) / L_{\text {mic }}(p, 100)\right]$ as a function of annealing time for the different ratios of the mass of unimer added to the hot seed solution, $m_{\text {uni,added }}$, to the mass of seeds originally present in the solution, $\mathrm{m}_{\text {seeds,RT }}(p=0,0.9,2,3.1,4.5,4.9)$.

\subsection{Fragmentation versus Annealing Time}

The presence of fragmentation could strongly affect the micelle growth kinetics. Indeed, the impact of fragmentation on the micelle lengths during seeded growth is multifold. It decreases the length of the micelles that fragment, and it increases the number of seeds the unimer can add on. Fragmentation could also influence the growth kinetics. For example, if seeded growth was a diffusion limited process, the increase in the number of seeds in solution would decrease the diffusion time between the unimer and the seed ends, which would lead to an increase in growth kinetics. It might thus appear difficult to quantify the effect of fragmentation on micelle growth.

Fortunately, we can use some key observations that were made in previous reports as well as in the present work to simplify the equations. Boott et al. [11] have shown that the seeded growth of $\mathrm{PFS}_{63}-b$-PDMS 513 (a system similar to that presented in this study) was not diffusion-limited. We can thus assume that the growth kinetics is not influenced by the seed fragmentation. In addition, the overlapping plots of $L_{\text {mic }}(p, t) / L_{\text {mic }}(p, 100)$ indicate that micelle fragmentation does not depend on the amount of unimer present in solution. Finally, we distinguish dissolution from fragmentation, in the sense that fragmentation leads to the formation of more micelles/seeds, but does not add any unimer in solution.

The number average length of a population of $N$ seeds is given by:

$$
L_{n}=\frac{\sum_{i=1}^{N} L_{i}}{N}
$$


where $L_{i}$ is the length of seed $i$, and $N$ is the total number of seeds. If $z$ new seeds are formed via fragmentation, the number average length of the seed solution, $L_{n, f}$ becomes:

$$
L_{t s, f}(t)=\frac{\sum_{i=1}^{N+f} L_{i}^{\prime}(t)}{N+z(t)}=\frac{\sum_{i=1}^{N} L_{i}}{N+z(t)}=\frac{\sum_{i=1}^{N} L_{i}}{N} \frac{1}{1+z(t) / N}=L_{t s}(0,0) f(t)
$$

where $L_{i}^{\prime}$ is the length of seed $i$, after fragmentation. It is important to note that Equation (10) is only correct in absence of dissolution during the fragmentation, since in this case, the total length of the seeds is unchanged and $z(t) / N$ increases with time, from 0 to a finite positive value.

In the present work, seed annealing can be schematized as a two-step process. First, as soon as the annealing temperature was reached, the seeds with the lowest crystallinity dissolved [36], while the rest of the seeds survived. The dissolution step can be considered instantaneous. In the second step, the seeds fragmented. As shown in Figures 2a, 3 and 4, this step is time dependent.

The difficulty here resides in choosing a physically meaningful equation that would describe the fragmentation of the trapped seeds during annealing. In a previous work [36], we showed that short seeds, such as those used in this study, would mainly fragment in the center until they reach a critical length, $L_{c, f}[40-43]$, that was estimated to be close to $32 \mathrm{~nm}$. Since we are using the same seed solution, we would expect the critical length to also be close to $32 \mathrm{~nm}$.

It is also important to note that the increase in length of the seeds during annealing at $75^{\circ} \mathrm{C}$ (Figure 2c,d), did not affect their fragmentation, since the plots of $L_{\text {mic }}(p, t) / L_{\text {mic }}(100, t)$ versus time (Figure 4 ) overlap for all the values of $p$. This result, in apparent contradiction with previous observations [44], suggests that the original seeds were more fragile than their extended counterparts. This phenomenon may find its origin in the fact that the original seeds were grown at room temperature, while the extended parts were grown at $75^{\circ} \mathrm{C}$, which could facilitate a better packing of the crystalline block, strengthening the micelle core.

From this description, we infer that the evolution of the number of fragmentation events as a function of time could be approximated by a normal distribution centered at $t=0$. Indeed, at short time, the number of seeds that would fragment might be relatively large, but as the annealing time increases, the seeds would strengthen, and the number of fragmentation events would slowly decrease. Since the number of seeds increases at each fragmentation event, one needs to consider the sum of all the events as a function of time, leading to a cumulative distribution function:

$$
z(t)=2 \int_{0}^{t} \frac{\exp \left(-\frac{x^{2}}{2 \sigma^{2}}\right)}{\sqrt{2 \pi \sigma^{2}}} d x[\alpha-1] N
$$

and

$$
f(t)=\frac{1}{1+z(t) / N}=\frac{1}{1+2 \int_{0}^{t} \frac{\exp \left(-\frac{x^{2}}{2 \sigma^{2}}\right)}{\sqrt{2 \pi \sigma^{2}}} d x[\alpha-1]}
$$

where $\sigma$ can be related to the rate of fragmentation, since fast fragmentation would lead to a low value of $\sigma$, while $\alpha$ is a normalization factor defined as:

$$
\alpha=\frac{L_{t s}(0,0)}{L_{c, f}}
$$

If the length of the surviving seeds at $t=0$ is equal to the critical length, $L_{c, f}$, then $\alpha=1$, and the seeds will not fragment. However, if $\alpha$ is large, then the decrease in seed lengths due to fragmentation will also be large. 
From Equation (10), the change of the trapped seed lengths as a function of fragmentation is thus:

$$
L_{t s, f}(0, t)=\frac{L_{t s}(0,0)}{2 \int_{0}^{t} \frac{\exp \left(-\frac{x^{2}}{2 \sigma^{2}}\right)}{\sqrt{2 \pi \sigma^{2}}} d x[\alpha-1]+1}
$$

Since the amount of unimer added to the seed solution did not affect their fragmentation, we can write the more general equation:

$$
L_{t s, f}(p, t)=\frac{L_{t s}(0,0)}{2 \int_{0}^{t} \frac{\exp \left(-\frac{x^{2}}{2 \sigma^{2}}\right)}{\sqrt{2 \pi \sigma^{2}}} d x[\alpha-1]+1}
$$

with $L_{t s}(0,0)=L_{t s}(p, 0)$, since at time $t=0$, no unimer would have time to add onto the surviving seeds.

Incorporating Equation (15) into Equation (7) leads to:

$$
L_{\text {mic }}(p, t)=(p+1) \frac{L_{\text {mic }}(0,0)}{2 \int_{0}^{t} \frac{\exp \left(-\frac{x^{2}}{2 \sigma^{2}}\right)}{\sqrt{2 \pi \sigma^{2}}} d x\left(\frac{L_{t s}(0,0)}{L_{c, f}}-1\right)+1}
$$

Equation (16) can thus be used to fit $L_{m i c}(p, t) /(1+p)$ versus time (Figure $\left.5 a\right)$. For this fit, we used the values of $\sigma=2700 \mathrm{~min}, L_{m i c}(0,0)=65 \mathrm{~nm}, L_{t s}(0,0)=41 \mathrm{~nm}$ and $L_{c, f}=32 \mathrm{~nm}$, which is the value expected from the superblob approach [36]. In a previous work, we have shown that in dilute solution seeds dissolve in a cooperative (explosive) process. Thus, seeds that survive dissolution would be expected to have a length similar to the original seeds, i.e., $43.5 \mathrm{~nm}$, close to the value of $L_{t s}(0,0)=41 \mathrm{~nm}$ used to fit the data.

Fits of $L_{m i c}(p, t)$ as a function of $p$ and $t$ are shown in Figure $5 b, c$. Despite the inherent uncertainty in the measurements of the micelle lengths, we could fit reasonably well the experimental data shown in Figure 5b,c using Equation (16).

\subsection{Growth Kinetics at $75^{\circ} \mathrm{C}$}

Equation (16) gives the evolution of the number average lengths of the micelles once they were fully regrown at room temperature. The lengths of these micelles were only dependent on the number of seeds present in solution, i.e., seed fragmentation. Growth kinetics could thus be ignored.

The situation is quite different for the seeds trapped at $75^{\circ} \mathrm{C}$, since the lengths of the micelles as a function of time depend on both micelle fragmentation and growth kinetics. Figure 2c,d, shows that the trapped seeds annealed for $2640 \mathrm{~min}$ were twice longer when $p=4.9$ than in absence of unimer added to the solution $(p=0)$. The master curve obtained for $L_{\text {mic }}(p, t) /(p+1)$ versus time (Figure $5 a$ ), however, suggests that fragmentation did not depend on the amount of unimer added. From these two observations, we conclude that fragmentation and micelles growth were independent from each other, in our experimental conditions. Indeed, if fragmentation was related to micelle growth, then a larger amount of micelles would have fragmented when the micelles were longer, and $L_{m i c}(p, t) /(p+1)$ would have varied with $p$. Therefore, fragmentation and growth can be seen as two independent time functions. We can thus write:

$$
L_{t s}(p, t)=f(t) g(p, t)
$$

where $f(t)$ is the fragmentation function given in Equation (12), while $g(p, t)$ describes micelle growth as a function of $p$ in absence of fragmentation:

$$
g(p, t)=\left[\frac{m_{u n i}(p, 0)-m_{u n i}(p, t)}{m_{t s}(p, 0)}+1\right] L_{t s}(p, 0)
$$


with $L_{t s}(p, 0)=L_{t s}(0,0)$, and $m_{t s}(p, 0)=m_{t s}(0,0)$.
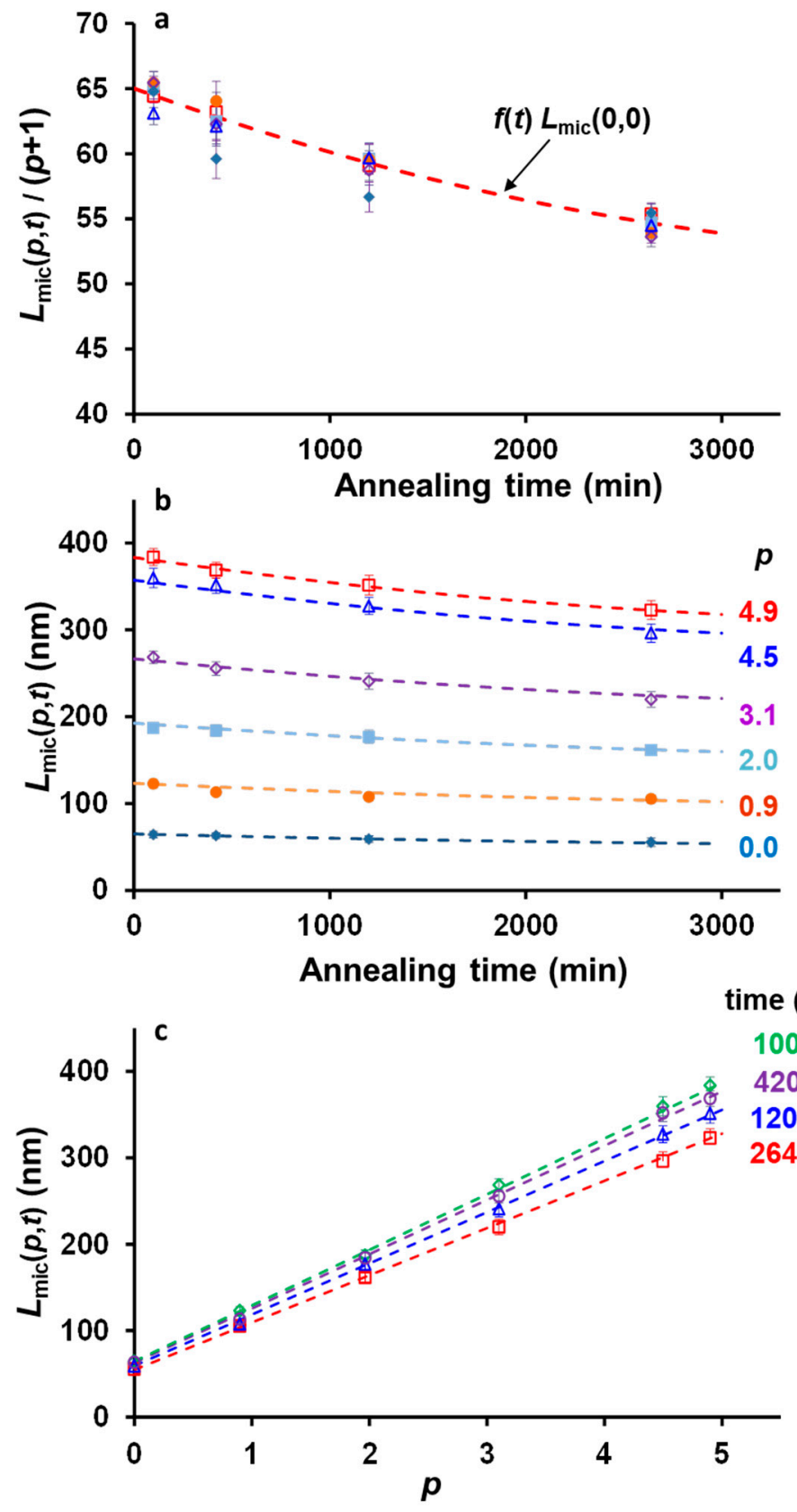

Figure 5. (a) Plot of $\left[L_{\text {mic }}(p, t) /(1+p)\right]$ as a function of annealing time (symbols). Fitting Equation (16) to these data (dashed line) led to the values of $\sigma=2700 \mathrm{~min}, L_{m i c}(0,0)=65 \mathrm{~nm}, L_{t s}(0,0)=41 \mathrm{~nm}$ and $L_{c, f}=32 \mathrm{~nm}$. Plots of the length of the regrown micelles, $L_{\text {mic }}(p, t)$, fitted to Equation (16) (dashed lines) as a function of (b) time and (c) $p$. Error bars correspond to the s.e.m. of the length distributions.

As shown in Supporting Information, taking into account the effect of the molecular weight distribution of $\mathrm{PFS}_{53}-b-\mathrm{PI}_{637}$, leads to:

$$
g(p, t)=L_{t s}(0,0)\left[1+\left(\frac{L_{m i c}(0,0)}{L_{t s}(0,0)}(1+p)-1\right)\left(1-e^{-\left(k^{*} t\right)^{\beta}}\right)\right]
$$

where $k^{*}$ is the growth rate constant and $\beta$, the stretching exponent.

A stretched exponential is a signature of a distribution of growth rates $[45,46]$. This kind of distribution could arise if the deposition rate of a unimer were highly sensitive either 
to the length of its PFS block or the block ratio of the BCP. Even though $\mathrm{PFS}_{53}-b-\mathrm{PI}_{637}$ has a narrow molecular weight distribution $(Đ=1.01)$, it is not monodispersed. The stretched exponential fit suggests that fractionation affects the micelle growth at $75^{\circ} \mathrm{C}$ [47]. This result is consistent with the observation made in one of the rare studies of the fractionation of narrowly dispersed BCP by crystallization [48]. It is also in agreement with the recent work from Song et al., who studied the CDSA of crystalline-coil BCPs with corona-forming block of various molecular weight distributions [49].

Incorporating Equations (16) and (19) into Equation (17) finally gives the growth kinetics of the micelles at $75^{\circ} \mathrm{C}$ in the presence of fragmentation.

$$
L_{t s}(p, t)=\frac{L_{t s}(0,0)\left[1+\left(\frac{L_{m i c}(0,0)}{L_{t s}(0,0)}(1+p)-1\right)\left(1-e^{-\left(k^{*} t\right)^{\beta}}\right)\right]}{2 \int_{0}^{t} \frac{\exp \left(-\frac{x^{2}}{2 \sigma^{2}}\right)}{\sqrt{2 \pi \sigma^{2}}} d x\left(\frac{L_{t s}(0,0)}{L_{c, f}}-1\right)+1}
$$

Fits of $L_{t s}(p, t)$ as a function of $p$ and $t$ are shown in Figure 6. To fit the growth kinetics data (Figure $6 \mathrm{a}, \mathrm{b}$ ), we used $k^{*}=1.7 \times 10^{-5} \mathrm{~min}^{-1}$ and $\beta=0.51$. Interestingly, these values compared well with the data obtained by Boott et al. [11] for the growth of $\mathrm{PFS}_{63}-b$-PDMS 513 in $n$-hexane at different temperatures. Indeed, extrapolation of the Eyring plot $\left(\ln \left(k^{\prime} / \mathrm{T}\right)\right.$ versus $1 / \mathrm{T}$ ) that they generated from kinetic data obtained at different temperatures led to a rate constant $k^{\prime}=4.4 \times 10^{-5} \mathrm{~min}^{-1}$ (or $k^{\prime}=7.3 \times 10^{-7} \mathrm{~s}^{-1}$ ), which is in the same order of magnitude as the rate constant deduced from Equation (20).

\subsection{Evaluation of the Growth Rate as a Function of Unimer Concentration}

Equation (20) gives us the possibility to evaluate the growth rate of the core crystalline micelles as a function of unimer concentration:

$$
G(p, t)=\frac{\partial L_{t s}(p, t)}{\partial t}
$$

By itself, $G(p, t)$ is extremely complex. We are, however, interested in evaluating the variation of $G(p, t)$ as a function of $p$. For this reason, one simply needs to rewrite Equation (20) to isolate all the terms that depend on $p$ :

$$
L_{t s}(p, t)=\frac{L_{t s}(0,0) e^{-\left(k^{*} t\right)^{\beta}}+L_{m i c}(0,0)\left(1-e^{-\left(k^{*} t\right)^{\beta}}\right)}{f(t)}+p \frac{L_{m i c}(0,0)\left(1-e^{-\left(k^{*} t\right)^{\beta}}\right)}{f(t)}=x(t)+p y(t)
$$

Therefore $G(p, t)$ is given by:

$$
G(p, t)=\frac{d x(t)}{d t}+p \frac{d y(t)}{d t}
$$

$G(p, t)$ thus increases linearly with $p$, and since $p$ is proportional to the unimer concentration, $c$, we can write that $G \propto c^{\gamma}$, with $\gamma=1$. This special case indicates that the micelle growth proceeded in the mononucleation regime, where one nucleus adds at a time on the growth face. The linear increase of $G(p, t)$ with $p$ is in agreement with the results of Monte Carlo simulations reported by $\mathrm{Hu}$ et al. $[50,51]$ for planar growth of $1 \mathrm{D}$ crystals. 

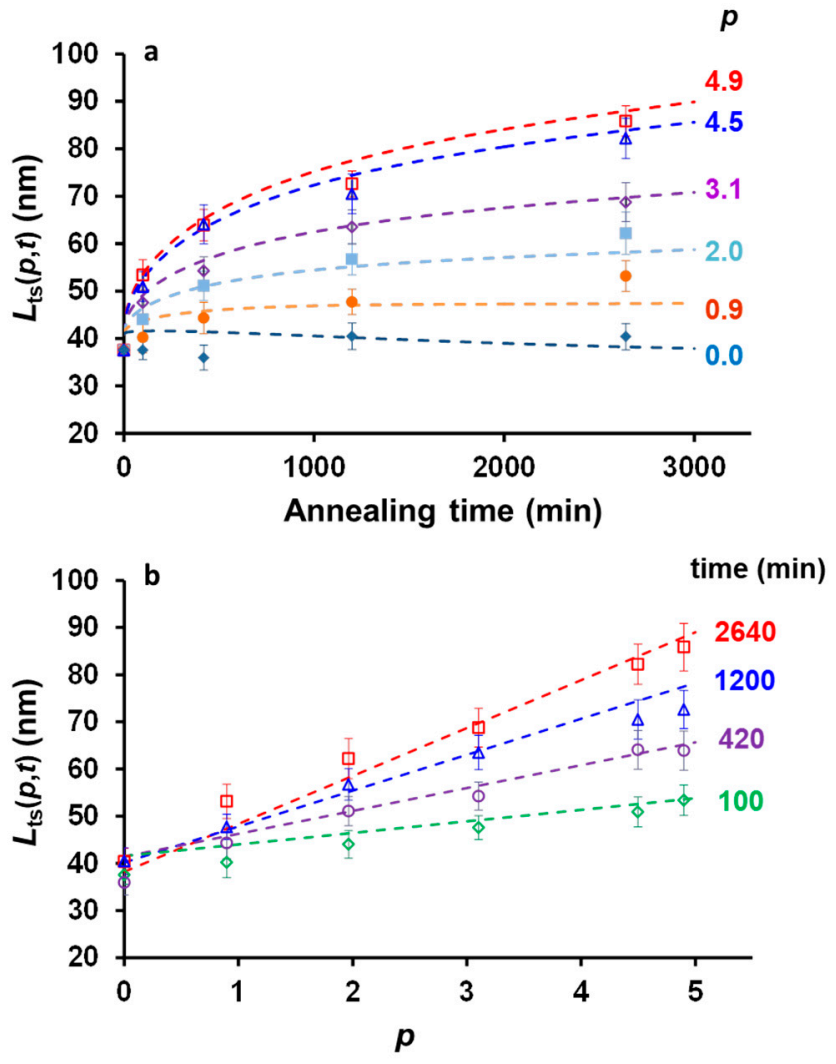

Figure 6. Plot of the of the length of the trapped crystallites, $L_{t s}(p, t)$ fitted to Equation (20) as a function of (a) time, and (b) $p$.

\section{Conclusions}

In summary, we have demonstrated that a seed-trapping protocol can be used to investigate the crystal growth kinetics of 1D micelles at elevated temperatures where both seed dissolution and fragmentation happened. Seed trapping proved particularly efficient in delineating the surviving seeds, allowing us to measure them after different annealing time. By comparing the length of the trapped seeds with the lengths of micelles that followed the exact same thermal history without being trapped, we could develop a kinetic model that accounted for seed fragmentation during crystallite growth. We considered that the probability that a seed fragment was decreasing with time, following a normal distribution function. The increase in number of seeds in solution could thus be described by a cumulative distribution function that is not dependent on the amount of unimer present in solution, and thus on the seeded growth kinetics. As previously reported, we observed that the seeded growth kinetics could be well modeled by a stretched exponential, which we believe is due to the fractionation by crystallization of the narrowly dispersed BCP [52]. Finally, we used our growth kinetics model to evaluate the variation of the growth rate as a function of the amount of unimer present in solution. We found that the $1 \mathrm{D}$ growth of the crystallites occurs in the mononucleation regime.

This study shows how seed trapping can be applied to study 1D micelle growth of other crystalline-coil BCPs, as well as more complex systems such as the parallel growth of two or three 1D crystals from a single BCP crystal face [53].

Supplementary Materials: The following are available online at https: / www.mdpi.com/article/ $10.3390 /$ polym13183122/s1. Supporting discussion Section I: Effect of lateral growth on $L_{\text {mic }}(p, t)$ during annealing. Supporting discussion Section II: Effect of fragmentation on $L_{\text {mic }}(p, t)$ during annealing. Supporting discussion Section III: Growth kinetics at $75^{\circ} \mathrm{C}$. Table S1: Values of $L_{\text {mic }}(p, t)$ of the $\mathrm{PFS}_{53}-b-\mathrm{PI}_{637}$ crystallites heated for different annealing times at $75{ }^{\circ} \mathrm{C}$ and cooled to room temperature (control experiments), Table S2: Values of $L_{\mathrm{ts}}(p, t)$ of the $\mathrm{PFS}_{53}-b-\mathrm{PI}_{637}$ crystallites heated 
for different annealing time at $75{ }^{\circ} \mathrm{C}$ (seed-trapping experiments), Figure S1: Effect of PFS53-b-PI637 seed crystallite history on their stability at RT, Figure S2: Effect of PFS53-b-PI637 seed crystallite history on their stability against dissolution upon heating at $75{ }^{\circ} \mathrm{C}$, Figure S3: Comparison of the histograms of the length distribution PFS53-b-PI637 seed crystallites that were trapped with $\mathrm{PFS}_{60^{-}}$ $b$-PDMS 660 unimer after being heated at $75^{\circ} \mathrm{C}$, Figure S4: TEM micrographs of PFS $_{53}-b$-PI 637 seeds trapped after ((a) $100 \mathrm{~min}$, ((b) $420 \mathrm{~min}$, ((c) $1200 \mathrm{~min}$ and (d) $2640 \mathrm{~min}$ of annealing at $75^{\circ} \mathrm{C}$ without $\mathrm{PFS}_{53}-b-\mathrm{PI}_{637}$ unimer added $(p=0)$, Figure S5: TEM micrographs of $\mathrm{PFS}_{53}-b-\mathrm{PI}_{637}$ seeds trapped after (a) $100 \mathrm{~min}$, (b) $420 \mathrm{~min}$, (c) $1200 \mathrm{~min}$ and (d) $2640 \mathrm{~min}$ of annealing at $75{ }^{\circ} \mathrm{C}$ in the presence of an initial mass ratio of $\mathrm{PFS}_{53}-b-\mathrm{PI}_{637}$ unimer added to $\mathrm{PFS}_{53}-b-\mathrm{PI}_{637}$ seeds $p=0.9$, Figure S6: TEM micrographs of $\mathrm{PFS}_{53}-b-\mathrm{PI}_{637}$ seeds trapped after ((a) $100 \mathrm{~min}$, ((b) $420 \mathrm{~min}$, (c) $1200 \mathrm{~min}$ and ((d) 2640 min of annealing at $75^{\circ} \mathrm{C}$ in the presence of an initial mass ratio of $\mathrm{PFS}_{53}-b-\mathrm{PI}_{637}$ unimer added to $\mathrm{PFS}_{53}-b-\mathrm{PI}_{637}$ seeds $p=2$, Figure S7: TEM micrographs of $\mathrm{PFS}_{53}-b-\mathrm{PI}_{637}$ seeds trapped after ((a) $100 \mathrm{~min},\left((\mathrm{~b}) 420 \mathrm{~min},\left((\mathrm{c}) 1200 \mathrm{~min}\right.\right.$ and (d() $2640 \mathrm{~min}$ of annealing at $75{ }^{\circ} \mathrm{C}$ in the presence of an initial mass ratio of $\mathrm{PFS}_{53}-b$ - $\mathrm{PI}_{637}$ unimer added to $\mathrm{PFS}_{53}-b$-PI 637 seeds $p=3.1$, Figure S8: TEM micrographs of $\mathrm{PFS}_{53}-b-\mathrm{PI}_{637}$ seeds trapped after ((a) $100 \mathrm{~min}$, ((b) $420 \mathrm{~min}$, (c) $1200 \mathrm{~min}$ and (d) $2640 \mathrm{~min}$ of annealing at $75{ }^{\circ} \mathrm{C}$ in the presence of an initial mass ratio of $\mathrm{PFS}_{53}-b$ - $\mathrm{PI}_{637}$ unimer added to $\mathrm{PFS}_{53}-b-\mathrm{PI}_{637}$ seeds $p=4.5$, Figure S9: TEM micrographs of $\mathrm{PFS}_{53}-b-\mathrm{PI}_{637}$ seeds trapped after (a) $100 \mathrm{~min}$, (b) $420 \mathrm{~min}$, (c) $1200 \mathrm{~min}$ and (d) $2640 \mathrm{~min}$ of annealing at $75^{\circ} \mathrm{C}$ in the presence of an initial mass ratio of $\mathrm{PFS}_{53}-b-\mathrm{PI}_{637}$ unimer added to $\mathrm{PFS}_{53}-b-\mathrm{PI}_{637}$ seeds $p=4.9$, Figure S10: Histograms of the length distributions of $\mathrm{PFS}_{53}-b-\mathrm{PI}_{637}$ trapped seeds after (a) $100 \mathrm{~min}$, (b) $420 \mathrm{~min}$, (c) $1200 \mathrm{~min}$ and (d) $2640 \mathrm{~min}$ of annealing at $75{ }^{\circ} \mathrm{C}$ without $\mathrm{PFS}_{53}-b-\mathrm{PI}_{637}$ unimer added $(p=0)$, Figure S11: Histograms of the length distributions of $\mathrm{PFS}_{53}-b-\mathrm{PI}_{637}$ trapped seeds after (a) $100 \mathrm{~min}$, (b) $420 \mathrm{~min}$, (c) $1200 \mathrm{~min}$ and (d) $2640 \mathrm{~min}$ of annealing at $75^{\circ} \mathrm{C}$ in the presence of an initial mass ratio of $\mathrm{PFS}_{53}-b-\mathrm{PI}_{637}$ unimer added to $\mathrm{PFS}_{53}-b-\mathrm{PI}_{637}$ seeds $p=1$, Figure S12: Histograms of the length distributions of $\mathrm{PFS}_{53}-b-\mathrm{PI}_{637}$ trapped seeds after (a) $100 \mathrm{~min}$, (b) $420 \mathrm{~min}$, (c) $1200 \mathrm{~min}$ and (d) $2640 \mathrm{~min}$ of annealing at $75{ }^{\circ} \mathrm{C}$ in the presence of an initial mass ratio of $\mathrm{PFS}_{53}-b$ - $\mathrm{PI}_{637}$ unimer added to $\mathrm{PFS}_{53}-b$-PI 637 seeds $p=2$, Figure S13: Histograms of the length distributions of $\mathrm{PFS}_{53}-b$ - $\mathrm{PI}_{637}$ trapped seeds after (a) $100 \mathrm{~min}$, (b) $420 \mathrm{~min}$, (c) $1200 \mathrm{~min}$ and (d) $2640 \mathrm{~min}$ of annealing at $75^{\circ} \mathrm{C}$ in the presence of an initial mass ratio of $\mathrm{PFS}_{53}-b-\mathrm{PI}_{637}$ unimer added to $\mathrm{PFS}_{53}-b-\mathrm{PI}_{637}$ seeds $p=3$, Figure S14: Histograms of the length distributions of $\mathrm{PFS}_{53}-b-\mathrm{PI}_{637}$ trapped seeds after (a) $100 \mathrm{~min}$, (b) $420 \mathrm{~min}$, (c) $1200 \mathrm{~min}$ and (d) $2640 \mathrm{~min}$ of annealing at $75^{\circ} \mathrm{C}$ in the presence of an initial mass ratio of $\mathrm{PFS}_{53}-b-\mathrm{PI}_{637}$ unimer added to $\mathrm{PFS}_{53}-b-\mathrm{PI}_{637}$ seeds $p=4.5$, Figure S15: Histograms of the length distributions of $\mathrm{PFS}_{53}-b-\mathrm{PI}_{637}$ trapped seeds after (a) $100 \mathrm{~min}$, (b) $420 \mathrm{~min}$, (c) $1200 \mathrm{~min}$ and (d) $2640 \mathrm{~min}$ of annealing at $75{ }^{\circ} \mathrm{C}$ in the presence of an initial mass ratio of $\mathrm{PFS}_{53}-b$ - $\mathrm{PI}_{637}$ unimer added to $\mathrm{PFS}_{53}-b-\mathrm{PI}_{637}$ seeds $p=4.8$, Figure S16: Histograms of the length of $\mathrm{PFS}_{53}-b-\mathrm{PI}_{637}$ seed crystallites in decane that were trapped with added $\mathrm{PFS}_{60}-b$-PDMS 660 unimer after being heated at $75^{\circ} \mathrm{C}$, then cooled to RT and stained with Karstedt's catalyst, Figure S17. Evolution of the number average lengths of the number average lengths of trapped seeds as a function of time for $p=0$, $0.9,2,3.1,4.5$ and 4.9, Figure S18: Evolution of the number average lengths of $\mathrm{PFS}_{53}-b-\mathrm{PI}_{637}$ seed crystallites annealed in decane at $75{ }^{\circ} \mathrm{C}$, and cooled to $23{ }^{\circ} \mathrm{C}$, as a function of the annealing time (control experiment), Figure S19: Plot of $L_{\text {mic }}(p, t)$ as a function of $(1+p) L_{\text {mic }}(100, t)$.

Author Contributions: G.G. conceived the project, performed the experiments. P.A.R. synthesized the polymers. G.G. and M.A.W. prepared the manuscript. The whole project was supervised by M.A.W. All authors have read and agreed to the published version of the manuscript.

Funding: This research was funded by the Science and Technology Commission of Shanghai Municipality, China (21ZR1415400).

Institutional Review Board Statement: Not applicable.

Informed Consent Statement: Not applicable.

Acknowledgments: G.G. thanks Sepehr Mastour Tehrani for his helpful comments. G.G. and M.A.W. thank Anton Zilman for their valuable comments and suggestions.

Conflicts of Interest: The authors declare no competing financial interests. 


\section{References}

1. Keller, A. A note on single crystals in polymers: Evidence for a folded chain configuration. Philos. Mag. 1957, 2, 1171-1175. [CrossRef]

2. Storks, K.H. An Electron Diffraction Examination of Some Linear High Polymers. J. Am. Chem. Soc. 1938, 60, 1753-1761. [CrossRef]

3. Bassett, D.C.; Keller, A. On the habits of polyethylene crystals. Philos. Mag. 1962, 7, 1553-1584. [CrossRef]

4. Lotz, B.; Kovacs, A.J.; Bassett, G.A.; Keller, A. Properties of copolymers composed of one poly-ethylene-oxide and one polystyrene block. Kolloid-Z. Z. Polym. 1966, 209, 115-128. [CrossRef]

5. Zhang, B.; Chen, J.; Baier, M.C.; Mecking, S.; Reiter, R.; Mülhaupt, R.; Reiter, G. Molecular-Weight-Dependent Changes in Morphology of Solution-Grown Polyethylene Single Crystals. Macromol. Rapid Commun. 2015, 36, 181-189. [CrossRef]

6. Xu, J.; Ma, Y.; Hu, W.; Rehahn, M.; Reiter, G. Cloning polymer single crystals through self-seeding. Nat. Mater. 2009, 8, 348-353. [CrossRef]

7. Zhang, B.; Chen, J.; Zhang, H.; Baier, M.C.; Mecking, S.; Reiter, R.; Mülhaupt, R.; Reiter, G. Annealing-induced periodic patterns in solution grown polymer single crystals. RSC Adv. 2015, 5, 12974-12980. [CrossRef]

8. Blundell, D.J.; Keller, A. The concentration dependence of the linear growth rate of polyethylene crystals from solution. J. Polym. Sci. Part B 1968, 6, 433-440. [CrossRef]

9. Dosiere, M.; Colet, M.-C.; Point, J.J. An isochronous decoration method for measuring linear growth rates in polymer crystals. J. Polym. Sci. Part B 1986, 24, 345-356. [CrossRef]

10. Tian, M.; Dosière, M.; Hocquet, S.; Lemstra, P.J.; Loos, J. Novel Aspects Related to Nucleation and Growth of Solution Grown Polyethylene Single Crystals. Macromolecules 2004, 37, 1333-1341. [CrossRef]

11. Boott, C.E.; Leitao, E.M.; Hayward, D.W.; Laine, R.F.; Mahou, P.; Guerin, G.; Winnik, M.A.; Richardson, R.M.; Kaminski, C.F.; Whittell, G.R.; et al. Probing the Growth Kinetics for the Formation of Uniform 1D Block Copolymer Nanoparticles by Living Crystallization-Driven Self-Assembly. ACS Nano 2018, 12, 8920-8933. [CrossRef] [PubMed]

12. Petzetakis, N.; Dove, A.P.; O'Reilly, R.K. Cylindrical micelles from the living crystallization-driven self-assembly of poly(lactide)containing block copolymers. Chem. Sci. 2011, 2, 955-960. [CrossRef]

13. Schmelz, J.; Schedl, A.E.; Steinlein, C.; Manners, I.; Schmalz, H. Length Control and Block-Type Architectures in Worm-like Micelles with Polyethylene Cores. J. Am. Chem. Soc. 2012, 134, 14217-14225. [CrossRef]

14. Qian, J.; Li, X.; Lunn, D.; Gwyther, J.; Hudson, Z.; Kynaston, E.; Rupar, P.A.; Winnik, M.A.; Manners, I. Uniform, High Aspect Ratio Fiber-like Micelles and Block Co-micelles with a Crystalline $\pi$-Conjugated Polythiophene Core by Self-Seeding. J. Am. Chem. Soc. 2014, 136, 4121-4124. [CrossRef] [PubMed]

15. Mihut, A.M.; Drechsler, M.; Möller, M.; Ballauff, M. Sphere-to-Rod Transition of Micelles formed by the Semicrystalline Polybutadiene-block-Poly(ethylene oxide) Block Copolymer in a Selective Solvent. Macromol. Rapid Commun. 2010, 31, 449-453. [CrossRef] [PubMed]

16. Pitto-Barry, A.; Kirby, N.; Dove, A.; Oreilly, R. Expanding the scope of the crystallization-driven self-assembly of polylactidecontaining polymers. Polym. Chem. 2014, 5, 1427-1436. [CrossRef]

17. Lazzari, M.; Scalarone, D.; Vazquez-Vazquez, C.; López-Quintela, M.A. Cylindrical Micelles from the Self-Assembly of Polyacrylonitrile-Based Diblock Copolymers in Nonpolar Selective Solvents. Macromol. Rapid Commun. 2008, 29 , $352-357$. [CrossRef]

18. Crassous, J.J.; Schurtenberger, P.; Ballauff, M.; Mihut, A.M. Design of block copolymer micelles via crystallization. Polymer 2015, 62, A1-A13. [CrossRef]

19. Wang, X.; Guerin, G.; Wang, H.; Wang, Y.; Manners, I.; Winnik, M.A. Cylindrical Block Copolymer Micelles and Co-Micelles of Controlled Length and Architecture. Science 2007, 317, 644-647. [CrossRef]

20. He, F.; Gädt, T.; Jones, M.; Scholes, G.D.; Manners, I.; Winnik, M.A. Synthesis and Self-Assembly of Fluorescent Micelles from Poly(ferrocenyldimethylsilane- $b$-2-vinylpyridine- $b$-2,5-di(2'-ethylhexyloxy)-1,4-phenylvinylene) Triblock Copolymer. Macromolecules 2009, 42, 7953-7960. [CrossRef]

21. Nazemi, A.; Boott, C.; Lunn, D.; Gwyther, J.; Hayward, D.W.; Richardson, R.; Winnik, M.A.; Manners, I. Monodisperse Cylindrical Micelles and Block Comicelles of Controlled Length in Aqueous Media. J. Am. Chem. Soc. 2016, 138, 4484-4493. [CrossRef] [PubMed]

22. Jia, L.; Zhao, G.; Shi, W.; Coombs, N.; Gourevich, I.; Walker, G.C.; Guerin, G.; Manners, I.; Winnik, M.A. A design strategy for the hierarchical fabrication of colloidal hybrid mesostructures. Nat. Commun. 2014, 5, 3882. [CrossRef] [PubMed]

23. Jia, L.; Tong, L.; Liang, Y.; Petretic, A.; Guerin, G.; Manners, I.; Winnik, M.A. Templated Fabrication of Fiber-Basket Polymersomes via Crystallization-Driven Block Copolymer Self-Assembly. J. Am. Chem. Soc. 2014, 136, 16676-16682. [CrossRef]

24. Rupar, P.A.; Chabanne, L.; Winnik, M.A.; Manners, I. Non-Centrosymmetric Cylindrical Micelles by Unidirectional Growth. Science 2012, 337, 559-562. [CrossRef]

25. Qiu, H.; Hudson, Z.M.; Winnik, M.A.; Manners, I. Multidimensional hierarchical self-assembly of amphiphilic cylindrical block comicelles. Science 2015, 347, 1329-1332. [CrossRef]

26. Hudson, Z.; Lunn, D.; Winnik, M.A.; Manners, I. Colour-tunable fluorescent multiblock micelles. Nat. Commun. 2014, 5, 3372. [CrossRef] 
27. Guerin, G.; Cruz, M.; Yu, Q. Formation of 2D and 3D multi-tori mesostructures via crystallization-driven self-assembly. Sci. Adv. 2020, 6, eaaz7301. [CrossRef] [PubMed]

28. Jarrett-Wilkins, C.N.; Pearce, S.; Macfarlane, L.R.; Davis, S.A.; Faul, C.F.J.; Manners, I. Surface Patterning of Uniform 2D Platelet Block Comicelles via Coronal Chain Collapse. ACS Macro Lett. 2020, 9, 1514-1520. [CrossRef]

29. Armitstead, K.; Goldbeck-Wood, G.; Keller, A. Polymer crystallization theories. In Macromolecules: Synthesis, Order and Advanced Properties; Springer: Berlin/Heidelberg, Germany, 1992; pp. 219-312. ISBN 9783540544906.

30. Lauritzen, J.I.; Hoffman, J.D. Theory of formation of polymer crystals with folded chains in dilute solution. J. Res. Natl. Bur. Stand. Sect. A 1960, 64, 73-102. [CrossRef]

31. Hoffman, J.D.; Lauritzen, J.I.; Passaglia, E.; Ross, G.S.; Frolen, L.J.; Weeks, J.J. Kinetics of polymer crystallization from solution and the melt. Kolloid-Zeitschrift Zeitschrift Polymere 1969, 231, 564-592. [CrossRef]

32. Frank, F. Nucleation-controlled growth on a one-dimensional growth of finite length. J. Cryst. Growth 1974, 22, 233-236. [CrossRef]

33. Toda, A.; Kiho, H.; Miyaji, H.; Asai, K. A Kinetic Theory on the Growth Rate of Polymer Single Crystals. J. Phys. Soc. Jpn. 1985, 54, 1411-1422. [CrossRef]

34. Kundagrami, A.; Muthukumar, M. Continuum theory of polymer crystallization. J. Chem. Phys. 2007, 126, 144901. [CrossRef] [PubMed]

35. Keller, A.; Pedemonte, E. A study of growth rates of polyethylene single crystals. J. Cryst. Growth 1973, 18, 111-123. [CrossRef]

36. Guerin, G.; Rupar, P.A.; Manners, I.; Winnik, M.A. Explosive dissolution and trapping of block copolymer seed crystallites. Nat. Commun. 2018, 9, 1158. [CrossRef] [PubMed]

37. Guerin, G.; Molev, G.; Pichugin, D.; Rupar, P.A.; Qi, F.; Cruz, M.; Manners, I.; Winnik, M.A. Effect of Concentration on the Dissolution of One-Dimensional Polymer Crystals: A TEM and NMR Study. Macromolecules 2019, 52, 208-216. [CrossRef]

38. Guerin, G.; Molev, G.; Rupar, P.A.; Manners, I.; Winnik, M.A. Understanding the Dissolution and Regrowth of Core-Crystalline Block Copolymer Micelles: A Scaling Approach. Macromolecules 2020, 53, 10198-10211. [CrossRef]

39. Rupar, P.A.; Cambridge, G.; Winnik, M.A.; Manners, I. Reversible Cross-Linking of Polyisoprene Coronas in Micelles, Block Comicelles, and Hierarchical Micelle Architectures Using Pt(0)—Olefin Coordination. J. Am. Chem. Soc. 2011, 133, 16947-16957. [CrossRef]

40. Birshtein, T.; Borisov, O.; Zhulina, Y.; Khokhlov, A.; Yurasova, T. Conformations of comb-like macromolecules. Polym. Sci. USSR 1987, 29, 1293-1300. [CrossRef]

41. Panyukov, S.; Zhulina, E.; Sheiko, S.S.; Randall, G.; Brock, J.; Rubinstein, M. Tension Amplification in Molecular Brushes in Solutions and on Substrates. J. Phys. Chem. B 2009, 113, 3750-3768. [CrossRef]

42. Panyukov, S.V.; Sheiko, S.S.; Rubinstein, M. Amplification of Tension in Branched Macromolecules. Phys. Rev. Lett. 2009, 102, 148301. [CrossRef]

43. Park, I.; Nese, A.; Pietrasik, J.; Matyjaszewski, K.; Sheiko, S.S. Focusing bond tension in bottle-brush macromolecules during spreading. J. Mater. Chem. 2011, 21, 8448-8453. [CrossRef]

44. Qian, J.; Lu, Y.; Cambridge, G.; Guerin, G.; Manners, I.; Winnik, M.A. Polyferrocenylsilane Crystals in Nanoconfinement: Fragmentation, Dissolution, and Regrowth of Cylindrical Block Copolymer Micelles with a Crystalline Core. Macromolecules 2012, 45, 8363-8372. [CrossRef]

45. Johnston, D.C. Stretched exponential relaxation arising from a continuous sum of exponential decays. Phys. Rev. B 2006, 74, 184430. [CrossRef]

46. Choi, S.; Lodge, T.P.; Bates, F.S. Mechanism of Molecular Exchange in Diblock Copolymer Micelles: Hypersensitivity to Core Chain Length. Phys. Rev. Lett. 2010, 104, 047802. [CrossRef] [PubMed]

47. Point, J.J.; Colet, M.C.; Dosiere, M. Experimental criterion for the crystallization regime in polymer crystals grown from dilute solution: Possible limitation due to fractionation. J. Polym. Sci. Part B 1986, 24, 357-388. [CrossRef]

48. Lotz, B.; Kovacs, A.J. Propriétés Des Copolymères Biséquencés Polyoxyéthylène-Polystyrène. Kolloid-Z. Z. Polym. 1966, 209, 97-114. [CrossRef]

49. Song, S.; Zhou, H.; Manners, I.; Winnik, M.A. Block copolymer self-assembly: Polydisperse corona-forming blocks leading to uniform morphologies. Chem 2021. [CrossRef]

50. Zhou, Y.; Hu, W. Kinetic Analysis of Quasi-One-Dimensional Growth of Polymer Lamellar Crystals in Dilute Solutions. J. Phys. Chem. B 2013, 117, 3047-3053. [CrossRef]

51. Shu, R.; Zha, L.; Eman, A.A.; Hu, W. Fibril Crystal Growth in Diblock Copolymer Solutions Studied by Dynamic Monte Carlo Simulations. J. Phys. Chem. B 2015, 119, 5926-5932. [CrossRef]

52. Pennings, A.J. Fractionation of polymers by crystallization from solutions. II. J. Polym. Sci. Part C 1962, 16, 1799-1812. [CrossRef]

53. Qiu, H.; Gao, Y.; Du, V.A.; Harniman, R.; Winnik, M.A.; Manners, I. Branched Micelles by Living Crystallization-Driven Block Copolymer Self-Assembly under Kinetic Control. J. Am. Chem. Soc. 2015, 137, 2375-2385. [CrossRef] [PubMed] 